\title{
Surpresas com relação à política monetária e o mercado de capitais: evidências do caso brasileiro
}

\author{
WALTER GONÇALVES JUNIOR* \\ WILLIAM EID JUNIOR
}

Monetary policy surprises and capital markets: evidences from the Brazilian case. This article evaluates the effects that monetary policy actions exert on Brazilian stock market. By the measures defined to estimate the surprise caused by $\mathrm{Co}$ mitê de Política Monetária do Banco Central do Brasil (COPOM) decisions, it was verified that to a hypothetic unexpected $1 \%$ increase in the target rate is associated an $1.3 \%$ average fall of Bovespa Index. Additional tests did not show distinct reactions caused by direction decisions, neither evidences from relevant recent economic events or decision contexts having influences on the surprise responses.

Keywords: monetary policy; Bovespa; surprises; event study.

JEL Classification: G14; G18; E52.

\section{INTRODUÇÃO}

O crescimento e o desenvolvimento econômico de um país de forma ordenada e sustentável pressupõem uma série de requisitos; o mais evidente deles é a preservação do poder de compra de sua moeda com o controle da inflação. Para tanto, se faz necessária a presença de uma autoridade monetária, figura responsável por atingir este e outros fins ligados à estabilidade monetária.

No Brasil, esse papel institucional é desempenhado pelo Banco Central do Bra-

\footnotetext{
* Respectivamente: Doutorando e mestre em Administração pela FGV-EAESP e analista do Banco Central do Brasil. E-mail: walter.goncalves@gmail.com; Professor titular da FGV-EAESP - Escola de Administração de Empresas de São Paulo da Fundação Getúlio Vargas. E-mail: William.eid@fgv.br. As opiniões e pontos de vista expressos neste artigo, bem como erros e omissões nele contidos, são exclusivamente devidos a seus autores, não refletindo necessariamente a visão do Banco Central do Brasil. Submetido: Outubro 2008: Aprovado: Setembro 2009.
} 
sil que, dentre os instrumentos disponíveis para tanto, tem empregado mais comumente a taxa básica de juro (e operações de mercado aberto para sua concretização) como forma de obter os efeitos desejados que levam à estabilidade de preços.

Tendo-se em vista os impactos de ampla repercussão na economia brasileira que as decisões sobre a taxa básica promovem, o interesse por elas gerado é crescente, ocupando por vezes local de destaque na imprensa e na literatura acadêmica econômica. Sabidamente, essas mudanças produzem efeitos nas taxas de juros, câmbio, crédito, nas expectativas e também nos preços dos ativos, esperando-se com isso que, ao afetar a dinâmica destes canais, possa-se influenciar níveis de poupança, investimento e demanda agregada, o que em última instância afetaria a taxa de inflação.

No presente estudo, pretende-se lançar foco no último dos canais mencionados: o preço dos ativos. Atuando através das variações na riqueza dos agentes econômicos, antevê-se que quedas não previstas nas taxas de juros elevem os preços das ações, porque daquelas possivelmente decorreria um crescimento da economia, podendo levar a aumentos nos lucros e dividendos distribuídos pelas empresas, sem mencionar as menores taxas que os descontariam para a apropriação de valores de mercado; trata-se portanto de um canal muito relevante, especialmente em países como Estados Unidos e Inglaterra.

Constitui-se então em um interessante objeto de estudo observar-se como o mercado acionário brasileiro se comporta segundo os estímulos proporcionados pelas decisões do COPOM; pretende-se assim mensurar e analisar as respostas do mercado acionário às decisões do COPOM sobre a meta para a taxa básica de juro, tomando-se para tanto o índice agregado da Bolsa de Valores de São Paulo (Bovespa).

A relevância do tema é clara: sob a ótica da autoridade monetária, possuir estimativas confiáveis das reações dos mercados em seus diversos instrumentos é uma informação crítica para a formulação de suas políticas, bem como para a definição das decisões; pela perspectiva dos participantes do mercado financeiro, há um interesse igualmente acentuado — dada a influência exercida pela política monetária sobre as expectativas dos mercados, conhecer tais estimativas representaria uma relevante informação para a construção de posições e estratégias, tanto de investimento como de hedge, bem como para a gestão dos riscos implícitos às operações normalmente desenvolvidas em seu dia a dia.

\section{REVISÃO BIBLIOGRÁFICA}

Há uma ampla literatura para estudar os efeitos da política monetária, especialmente aqueles intervenientes nas estruturas das taxas de juros de curto prazo; Cook e Hahn (1989), constataram uma resposta intensa das taxas de curto prazo e fraca nas de longo prazo às mudanças nas taxas-alvo dos Fed Funds da década de 1970.

Roley e Sellon (1995), analisando as taxas de longo prazo, encontraram evi- 
dências que estas se antecipam às mudanças na política monetária e, em Roley e Sellon (1998a), estimaram respostas significativas das taxas de juros de longo prazo.

Há ainda diversos outros trabalhos que tratam da relação política monetária versus taxas de juros, frequentemente se valendo da abordagem por estudo de evento, tal como nos trabalhos retro referidos; em especial, Kuttner (2001) produziu um estudo similar, porém tentando isolar as componentes esperada e não esperada (ambas não diretamente observáveis) das mudanças nas taxas dos Fed Funds causadas por decisões do FOMC (Federal Open Market Committee). Ele encontrou uma relação robusta entre as surpresas com relação à política monetária e as taxas de juros de diferentes maturidades.

Outros estudos estabeleceram foco nos impactos da política monetária sobre o mercado acionário; Smirlock e Yawitz (1985) verificaram como incrementos exógenos nas taxas de redesconto reduziram os retornos calculados pelo índice NYSE na primeira metade da década de 1980.

Thorbecke e Alami (1994) constataram uma resposta significativa dos retornos das ações na segunda metade da década de 1970 (representadas pelos índices Dow Jones e S\&P) às mudanças na taxa-alvo pelo FOMC; Thorbecke (1997) ampliou este estudo usando diferentes abordagens (entre elas o estudo de evento) abrangendo o período de 1987 a 1994 (essencialmente quando a direção do Fed foi de Alan Greenspan), obtendo resultados qualitativamente similares aos de seu trabalho anterior.

Estudando um período mais restrito (de 1989 a 1992), mas com uma quantidade maior de instrumentos financeiros sob análise, Reinhart e Simin (1997) verificaram também tais respostas, ressalvando apenas que tais reações pareciam persistir por mais alguns dias além daquele subsequente à mudança, fato este explicado pela prática de então do Fed em não divulgar ao público suas decisões.

Outros trabalhos focando a relação entre mercado acionário e política monetária se seguiram, com abordagens e períodos similares; por vezes, obtiveram-se respostas significativas dos preços das ações à política monetária, como em Bomfim (2003), que também verificou um aumento anormal da volatilidade dos mercados em dias de reunião; por outro lado, como em Roley e Sellon (1998b) e Bomfim e Reinhart (2000), respostas não significativas também foram encontradas.

Artigos mais recentes como o de Rigobon e Sack (2004), que usaram estimadores para explorar a heterocedasticidade introduzida por ações de política monetária, ou o de Craine e Martin (2003), que desenvolveram um modelo de fatores multivariado para respostas dos ativos a choques de informação comuns e não observáveis, resultaram também em respostas significativas do mercado acionário às ações do $F e d$, com conclusões parecidas aos estudos de evento convencionais.

Bernanke e Kuttner (2005), a partir do recurso empregado por Kuttner (2001) para isolar a componente inesperada ou efeito surpresa nas mudanças da taxa-alvo dos Fed Funds, encontraram também respostas significativas e mais intensas dos preços das ações a esta componente - uma tentativa portanto de isolar, dentro das variações nas taxas-alvo, o que o mercado financeiro consegue antecipar (que pela 
hipótese dos mercados eficientes, não deveria causar respostas), daquilo que não é possível prever.

Neste sentido, Poole, Rasche e Thornton (2002) já haviam observado que estudos de evento como este utilizam especificações sujeitas a erros de medição nos regressores, havendo ainda problemas de correlação entre estes últimos e os erros; eles propuseram então mudanças que transformavam a análise em um problema típico de erros nas variáveis, obtendo respostas parecidas com os trabalhos anteriores e concluindo que tais estudos de evento geram resultados que, embora significativos, possivelmente subestimam a intensidade destas respostas.

Com relação ao observado no Japão, Honda e Kuroki (2006), conduzindo um estudo de evento para o período de 1989 a 2001, verificaram respostas significativas para a surpresa com relação às decisões do BOJ (Bank of Japan) sobre política monetária, tanto na estrutura a termo das taxas de juros quanto nos preços dos ativos, portanto resultados similares aos encontrados para os mercados norte-americanos.

No Brasil, através de um estudo de evento para o período de 1996 a 2003, Tabak e Tabata (2004a) não encontraram evidências de que a estrutura a termo de juro brasileira responda a ações de política monetária, medindo-se pelas mudanças na meta da taxa básica de juro do Banco Central; encontraram contudo um certo grau de antecipação dos mercados a tais ações, o que explicaria tais resultados. Posteriormente, ampliaram o âmbito de tal trabalho, decompondo a meta para a taxa básica de juro em meta antecipada e não antecipada; assim, Tabak e Tabata (2004b) em um novo estudo de evento para o período de 2000 a 2003, confirmaram o grau de antecipação anteriormente visto e constataram respostas significativas da estrutura a termo à componente não-antecipada.

Por sua vez, Gonçalves e Guimarães (2005), adotando a abordagem sugerida por Rigobon e Sack (2004) de explorar a heterocedasticidade introduzida pelas ações do Banco Central, usaram as taxas de juros anuais para verificar as respostas produzidas nas taxas do mercado de câmbio, encontrando que incrementos inesperados nos juros levaram a depreciações na taxa de câmbio, observando-se dados de 2000 a 2005, sob significâncias moderadas a fracas, conforme as subjanelas históricas selecionadas do período em questão.

Por fim, Robitaille e Roush (2005) empregaram estudos de evento adaptando as metodologias propostas por Kuttner (2001) e Gürkaynak (2005) a partir de dados intraday referenciados ao período de 1999 a 2004, obtendo respostas significativas tanto nos spreads dos C-Bonds brasileiros como no Índice Bovespa às decisões do FOMC e a alguns anúncios de indicadores macroeconômicos americanos, evidenciando assim o grau de relacionamento entre tais mercados (brasileiro e americano) e seus comportamentos.

\section{ESTIMANDO A SURPRESA DOS MERCADOS}

De modo geral, os mercados financeiros estão constantemente incorporando novas informações a seus preços; os agentes ditos racionais utilizam toda a infor- 
mação disponível em suas previsões, tomando valores passados e correntes de variáveis exógenas ou não (Lucas e Sargent, 1996).

Devido a essa característica dos mercados e em se tratando especificamente da Bolsa de Valores de São Paulo (onde Perobelli et al. (2000) identificaram uma eficiência informacional semiforte), verificar-se suas respostas às ações de política monetária pode ser uma tarefa particularmente difícil, porque cada decisão tomada pelo COPOM é estudada por esses agentes, que antecipam em alguma medida seus resultados; assim sendo, conforme observou Dewbre (1981), quaisquer mudanças verificadas nas variáveis correntes e futuras através do tempo decorrerão exclusivamente de mudanças não antecipadas.

Isto posto, ao analisarmos uma relação direta entre as variações na meta da taxa de juro e as do Índice Bovespa devemos encontrar baixa significância entre essas variáveis; isto leva, portanto, à necessidade de distinguir na variação da meta suas duas componentes principais: a antecipada e a não antecipada. Uma ferramenta interessante para se atingir tal intento é empregar a metodologia desenvolvida por Kuttner (2001), aplicada aos contratos futuros de taxa média de depósitos interbancários de um dia (referidos comumente por DI 1 dia), negociados na Bolsa de Mercadorias e Futuros de São Paulo (BM\&F).

Devemos, portanto, construir uma equação que, além de representar esta propriedade, ao mesmo tempo expresse adequadamente as idiossincrasias deste mercado; uma expressão adequada - a exemplo do adotado em Garcia e La Rocque (1994) — seria:

$$
\mathrm{PU}_{\mathrm{t}}=\mathrm{E}_{\mathrm{t}}\left(\frac{100000}{1+\mathrm{i}_{\mathrm{p}}}\right) \text {, onde: }
$$

$\mathrm{PU}_{\mathrm{t}}=$ Preço unitário do contrato DI 1 dia, em pontos, correspondente a 100.000, descontado pela taxa de juro implícita ao contrato;

$\mathrm{i}_{\mathrm{p}}=$ Taxa de juro esperada referente ao período considerado.

Se considerarmos que $\left(1+\mathrm{i}_{\mathrm{p}}\right)=\left(1+\overline{\mathrm{i}}_{\mathrm{m}}\right)^{t}$, onde $\overline{\mathrm{i}}_{\mathrm{m}}=\mathrm{E}_{\mathrm{t}}\left(\mathrm{i}_{\mathrm{m}}\right)$ é uma taxa média esperada ao dia útil para todo o períódo (até a liquidação do instrumento) e t é o número de dias úteis até a liquidação, então teremos:

$$
\mathrm{PU}_{\mathrm{t}}=\mathrm{E}_{\mathrm{t}}\left(\frac{100000}{1+\mathrm{i}_{\mathrm{p}}}\right)=\frac{100000}{\left(1+\overline{\mathrm{i}}_{\mathrm{m}}\right)^{\mathrm{t}}}
$$

Suporemos que no dia d-1 ocorre a reunião do COPOM e que no dia útil seguinte (que chamaremos de d) os mercados abrem já cientes da nova meta para a taxa básica de juro, já que em d ocorre a implantação da nova meta. Deste modo, em $\mathrm{d}-\mathbf{1}$ espera-se que a taxa média diária passe de $\frac{\mathrm{d}}{\mathrm{i}}$ (antes da reunião) para $\overline{\mathrm{i}}_{\mathrm{m}}$ (após a reunião) e que assim permaneça até a liquidação do instrumento; no entanto, em alguma medida eles são surpreendidos pelo COPOM e esta surpresa (que denominaremos $\Delta \mathbf{i}^{\mathrm{u}}$ ) passa a ser incorporada ao valor médio diário anteriormente previsto: 
os mercados se ajustam a eventuais discrepâncias em relação a suas projeções da véspera.

Portanto, empregando os contratos DI 1 dia com duração de 1 mês, teremos:

em d-1

(expectativas no dia da reunião)

$$
\frac{100000}{\mathrm{PU}_{\mathrm{d}-1}}=\left(1+\overline{\mathrm{i}}_{\mathrm{a}}\right) \cdot\left(1+\overline{\mathrm{i}}_{\mathrm{m}}\right)^{\mathrm{D}}
$$

em d

(expectativas no dia da implantação)

$$
\frac{100000}{P U_{d}}=\left(1+\overline{\mathrm{i}}_{\mathrm{m}}\right)^{\mathrm{D}} \cdot\left(1+\Delta \mathrm{i}^{\mathrm{u}}\right)^{\frac{\mathrm{D}}{252}}
$$

Da relação entre (4) e (3) obtemos:

$$
\left(1+\Delta \mathrm{i}^{\mathrm{u}}\right)=\left[\left(1+\overline{\mathrm{i}}_{\mathrm{a}}\right) \frac{\mathrm{PU}_{\mathrm{d}-1}}{\mathrm{PU}_{\mathrm{d}}}\right]^{\frac{252}{\mathrm{D}}}
$$

Para a obtenção de uma estimativa para $\bar{i}$, é preciso supor-se que na véspera do dia da reunião (antevéspera do dia do evento, portanto) as expectativas sejam similares às do dia em que ela ocorre; isto equivaleria a afirmar que $\mathrm{E}_{\mathrm{d}-2}\left(\mathrm{i}_{\mathrm{m}}\right) \cong \mathrm{E}_{\mathrm{d}-1}$ $\left(\mathrm{i}_{\mathrm{m}}\right)=\overline{\mathrm{i}}_{\mathrm{m}}$ e que $\mathrm{E}_{\mathrm{d}-2}\left(\mathrm{i}_{\mathrm{a}}\right) \cong \mathrm{E}_{\mathrm{d}-1}\left(\mathrm{i}_{\mathrm{a}}\right)=\overline{\mathrm{i}}_{\mathrm{a}}$.

Logo, $\frac{100000}{\mathrm{PU}_{\mathrm{d}-2}}=\left(1+\overline{\mathrm{i}}_{\mathrm{a}}\right)^{2} \cdot\left(1+\overline{\mathrm{i}}_{\mathrm{m}}\right)^{\mathrm{D}}$, a qual relacionada com (3) implica $\frac{\mathrm{PU}_{\mathrm{d}-1}}{\mathrm{PU}_{\mathrm{d} \cdot 2}}=\left(1+\overline{\mathrm{i}}_{\mathrm{a}}\right)$

Substituindo-se este resultado em (5), temos então:

$$
\left(1+\Delta \mathrm{i}^{\mathrm{u}}\right)=\left(\frac{\mathrm{PU}_{\mathrm{d}-1}^{2}}{\mathrm{PU}_{\mathrm{d}-2} \cdot \mathrm{PU}_{\mathrm{d}}}\right)^{\frac{252}{\mathrm{D}}}
$$

Portanto, (6) permite que estimemos a componente não prevista ou inesperada $\Delta \mathbf{i}^{u}$ pelos mercados na variação da meta da taxa básica de juro por decisão do COPOM. Esta medida apresenta, contudo, o inconveniente de necessitar de PUs negociados em até dois dias úteis anteriores ao dia do evento, o que constituiria um problema com dados de decisões em início de mês; esta incongruência, que ocorre em somente duas observações, será tratada em mais detalhes a seguir, na descrição dos dados.

A obtenção da componente esperada, consequentemente, virá de:

$$
\Delta \mathrm{i}^{\mathrm{e}}=\frac{(1+\Delta \mathrm{i})}{\left(1+\Delta \mathrm{i}^{\mathrm{u}}\right)}-1, \text { onde: }
$$


$\Delta \mathrm{i}=$ Variação na meta da taxa básica de juro por decisão do COPOM (base 252 dias úteis);

$\Delta \mathrm{i}^{\mathrm{e}}=$ Componente esperada da variação na meta da taxa básica de juro (base 252 dias úteis);

$\Delta \mathrm{i}^{\mathrm{u}}=$ Componente [não esperada da variação na meta da taxa básica de juro (252 dias úteis).]

Para conveniência nas especificações e testes a serem conduzidos doravante, os dados podem ser transformados para viabilizar o emprego do regime de capitalização contínua nas taxas. Logo, é possível fazer:

$\mathrm{I}^{\mathrm{u}}=\ln \left(1+\Delta \mathrm{i}^{\mathrm{u}}\right)$ : Surpresa ou componente não esperada (regime de capitalização contínua);

$\mathrm{I}^{\mathrm{e}}=\ln \left(1+\Delta \mathrm{i}^{\mathrm{e}}\right)$ : Componente esperada (regime de capitalização contínua);

I $=\ln (1+\Delta \mathrm{i})$ : Variação na meta da taxa básica (regime de capitalização contínua).

Note-se que, conforme (7) e segundo esta forma de tratar as taxas, temos que $\mathrm{I}^{\mathrm{u}}+\mathrm{I}^{\mathrm{e}}=\mathrm{I}$.

\section{RESPOSTAS DO MERCADO DE AÇÕES À POLÍTICA MONETÁRIA}

Trata-se portanto de um estudo de evento visando-se a análise do relacionamento entre as componentes citadas da variação da meta da taxa básica de juro e a variação do mercado entre os dois instantes selecionados para a captura da surpresa. Isso pode ser feito com a especificação:

$$
\Delta \mathrm{P}_{\mathrm{t}}=\alpha+\beta_{\mathrm{u}} \cdot \mathrm{I}_{\mathrm{t}}^{\mathrm{u}}+\beta_{\mathrm{e}} \cdot \mathrm{I}_{\mathrm{t}}^{\mathrm{e}}+\varepsilon_{\mathrm{t}}
$$

As hipóteses nulas a serem testadas são $\beta_{\mathrm{e}}=0$ e $\beta_{\mathrm{u}}=0$. Para tanto, temos que: $\Delta \mathrm{P}_{\mathrm{t}}=\mathrm{E}$ o logaritmo natural da razão entre cotações de fechamento do Índice Bovespa, tomando-se os dois pregões consecutivos correspondentes à mudança na meta para a taxa básica de juro;

$\mathrm{I}_{\varepsilon_{\mathrm{t}}}^{\mathrm{e}}=$ Componentes surpresa e esperada como supradefinido;

$\mathrm{I}_{\mathrm{t}}^{\mathrm{e}},=$ Termo de erro representativo de fatores outros não ligados à política monetária.

Seguindo aqui a mesma premissa adotada por Bernanke e Kuttner (2005), assumiremos a ortogonalidade do termo devido ao erro $\left(\varepsilon_{\mathrm{t}}\right)$ em relação às mudanças e surpresas assim calculadas, discutindo posteriormente as limitações e a viabilidade de tal assunção.

Será interessante também comparar os resultados da especificação (8) com 
uma outra que analise a resposta do mercado à variação da meta propriamente dita. Esta especificação seria:

$$
\Delta \mathrm{P}_{\mathrm{t}}=\alpha+\beta \cdot \mathrm{I}_{\mathrm{t}}
$$

Tendo $I_{t}$ já sido definida anteriormente.

Por fim, para evitar que os resultados sejam influenciados por observações que tenham impacto excessivamente pronunciado em relação às demais observações da amostra, é imprescindível identificá-las, promovendo-se então os testes a serem conduzidos com e sem a sua participação na amostra, explorando assim a robustez dos resultados. Bernanke e Kuttner (2005) sugeriram empregar a seguinte estimativa:

$$
\mathrm{IE}_{\mathrm{i}}=\Delta \beta_{\mathrm{i}}^{\mathrm{T}} \sum^{-1} \Delta \beta_{\mathrm{i}}
$$

Onde:

$\mathrm{IE}^{\mathrm{i}}=$ Influência estatística da observação i;

$\Delta \beta^{\mathrm{i}}=$ Mudança no vetor dos coeficientes da regressão (8) - ou seja, nos valores $\alpha, \beta^{\mathrm{u}}$ e $\beta^{\mathrm{e}}$ - resultante da exclusão da observação i da amostra;

$\Sigma=$ Matriz Variância-Covariância dos coeficientes da regressão (8).

Desta maneira, as observações cujas influências estatísticas apresentarem uma disparidade clara em relação às demais serão consideradas outliers; isso permitirá a realização dos dois ensaios distintos supramencionados: com e sem os outliers.

\section{QUEBRAS ESTRUTURAIS E ASSIMETRIA NAS RESPOSTAS}

O passo seguinte será investigar como alguns eventos relevantes na história econômica recente, tais como a adoção do câmbio flutuante, as taxas em expressão anual ou mesmo aspectos contextuais das decisões (se extraordinárias ou se tomadas em reuniões regulares) afetaram estas respostas. Outra possibilidade a ser explorada é se o mercado acionário reage distintamente conforme a direção tomada pela decisão do COPOM (elevação, manutenção, queda ou reversão da tendência anterior).

Estas questões podem ser estudadas a partir da introdução de variáveis dummy interativas com a surpresa, definidas para captar mudanças no comportamento das respostas conforme o aspecto a ser focado. A especificação genérica para conduzir tais testes será:

$$
\Delta \mathrm{P}_{\mathrm{t}}=\alpha+\beta_{\mathrm{u}} \cdot \mathrm{I}_{\mathrm{t}}^{\mathrm{u}}+\beta_{e} \cdot \mathrm{I}_{\mathrm{t}}^{\mathrm{e}}+\beta_{\mathrm{ef}} \cdot \text { DummyEfeito }_{\mathrm{t}} \cdot \mathrm{I}_{\mathrm{t}}^{\mathrm{u}}+\varepsilon_{\mathrm{t}}
$$

Onde DummyEfeito é a dummy interativa que assume valor igual a 1 quando o efeito ou evento estudado se verifica; caso contrário, assume valor zero. No presente trabalho serão utilizadas: 
- Flexibilização do câmbio: a dummy assume valor igual a 1 se a observação for posterior a 15/01/1999; caso contrário, assume valor zero;

- Adoção de taxas ano-over: a dummy assume valor igual a 1 se a observação for posterior a 02/01/1998; caso contrário, assume valor zero;

- Contexto das decisões: a dummy assume valor igual a 1 se a observação for resultante de uma reunião regular; caso contrário, assume valor zero;

- Aumento na meta: igual a 1 se a meta foi aumentada; caso contrário, assume valor zero;

- Redução na meta: igual a 1 se a meta foi reduzida; caso contrário, assume valor zero;

- Inatividade na meta: igual a 1 se a meta foi mantida sem alterações; caso contrário, assume valor zero;

- Reversão na meta: igual a 1 se houve uma reversão na tendência da meta (uma queda após uma sucessão de aumentos, ou o oposto); caso contrário, assume valor zero.

\section{DADOS}

O presente estudo depende, para suas estimativas, das variações na meta da taxa básica de juro, extraídas das Atas de Reunião disponíveis no website do Banco Central do Brasil; são utilizadas também as variações do Índice Bovespa, calculadas entre os dois instantes usados na obtenção das surpresas, a partir de suas cotações de fechamento diárias compreendidas entre junho de 1996 (início da sistemática de decisões de política monetária pelo COPOM) e março de 2006.

Totalizaram-se assim 125 observações, das quais a 17a (de 22/10/1997, época na qual se deu a crise dos países asiáticos) não entrou em vigor, tendo sido portanto excluída. Anteriormente a 02/01/1998, a prática do Banco Central era divulgar metas para a TBC em expressão mensal; no sentido de se manter a consistência dos valores na amostra, eles foram anualizados (base 252 dias úteis) a partir do número de dias úteis em que vigoraram.

As surpresas com relação a essas decisões foram calculadas a partir dos PUs de fechamento dos Contratos Futuros de Taxa Média de Depósitos Interfinanceiros de 1 dia com duração de um mês e de seus respectivos dias de saque, disponíveis no website da Bolsa de Mercadorias e Futuros de São Paulo (BM\&F). As cotações para o Índice Bovespa foram extraídas do banco de dados Economática ${ }^{\circledR}$, com as correções devidas.

A amostra assim constituída de componente esperada e não esperada para a mudança na meta da taxa básica de juro e das variações do Índice Bovespa possui, no entanto, algumas particularidades que devem ser mencionadas:

I. Há duas observações cujos dias de implantação da nova meta ocorrem nos dois primeiros dias úteis do mês: são as dos dias 05/04/1999 e 01/09/1999 (33a e 
38 a reuniões regulares). Estas são as duas situações que causam os conflitos mencionados em (6), conforme referido no item 3, que podem ser convenientemente contornados pela aplicação de (5) a regime de capitalização contínua, ou seja:

$$
I^{u}=\ln \left(1+\Delta i^{u}\right) \text {, onde }\left(1+\Delta i^{u}\right)=\left[\left(1+\bar{i}_{a}\right) \frac{P U_{d-1}}{P U_{d}}\right]^{252 / D}
$$

Podemos estimar $\bar{i}_{a}$ para estes dois casos a partir das taxas CDI - diferentemente de empregar os PUs, como fizemos nos demais casos, nestas duas situações não teremos todos os dados necessários em (6) referenciados ao mesmo mês; utilizar as taxas diárias CDI para estimar $\overline{\mathrm{i}}_{\mathrm{a}} \mathrm{e}$ um recurso que possibilitará a inclusão das observações em questão sem introduzir viés que comprometa os resultados.

II. Houve dez mudanças na meta (com reuniões ou não) consideradas extraordinárias, dado este obtido a partir de consulta ao agendamento pelo COPOM das reuniões seguintes mencionadas nas atas de reunião, como segue na Tabela 1 abaixo (as 114 restantes são consideradas regulares):

Tabela 1: Datas das reuniões ou mudanças não previstas em ata, com a variação na meta e o novo valor adotado

\begin{tabular}{|c|c|c|}
\hline Data Reunião & $\begin{array}{c}\text { Mudança } \\
\text { SELIC }\end{array}$ & $\begin{array}{c}\text { Nova Meta } \\
\text { p/ SELIC }\end{array}$ \\
\hline $10 / 09 / 1998$ & $0,00 \%$ & $19,00 \%$ \\
\hline $24 / 03 / 1999$ & $-3,00 \%$ & $42,00 \%$ \\
\hline $05 / 04 / 1999$ & $-2,50 \%$ & $39,50 \%$ \\
\hline $28 / 04 / 1999$ & $-2,00 \%$ & $32,00 \%$ \\
\hline $07 / 05 / 1999$ & $-2,50 \%$ & $29,50 \%$ \\
\hline
\end{tabular}

\begin{tabular}{|c|c|c|}
\hline Data Reunião & $\begin{array}{c}\text { Mudança } \\
\text { SELIC }\end{array}$ & $\begin{array}{c}\text { Nova Meta } \\
\text { p/ SELIC }\end{array}$ \\
\hline $12 / 05 / 1999$ & $-2,50 \%$ & $27,00 \%$ \\
\hline $08 / 06 / 1999$ & $-1,50 \%$ & $22,00 \%$ \\
\hline $28 / 03 / 2000$ & $-0,50 \%$ & $18,50 \%$ \\
\hline $07 / 07 / 2000$ & $-0,50 \%$ & $17,00 \%$ \\
\hline $14 / 10 / 2002$ & $3,00 \%$ & $21,00 \%$ \\
\hline
\end{tabular}

Em agosto de 1998 ocorreu a crise russa; neste período o Banco Central fazia uso da TBC (taxa básica de juro) e da TBAN (taxa de assistência para redesconto bancário) - a primeira, tida como piso para as taxas (frequente nas operações de captação do BC) e a segunda, como teto (mais comum na doação de recursos aos mercados).

Desse modo, as taxas variavam de acordo com a instituição na "banda" assim definida, conforme importância, garantias e risco dos tomadores; quando o cenário econômico se deteriorou, o Banco Central implantou, entre outras medidas, o aumento da TBAN com a manutenção da $\mathrm{TBC}$ - embora a procura maior por estes recursos tenha se dado (dentro e fora das operações de mercado aberto) nos níveis intermediários entre piso e teto, não raro as taxas se situaram mais próximas ao teto, função do ceticismo dos agentes quanto à reversão do quadro.

Iniciada em setembro de 1998, esta situação perdurou até novembro do mesmo ano, com a assinatura de um novo acordo do país com o Fundo Monetário 
Internacional (FMI) - assim, a Tabela 2 traz as quatro observações da amostra sujeitas ao caso:

Tabela 2: Datas das reuniões ligadas à crise russa

\begin{tabular}{|c|c|c|c|c|}
\hline Reunião & Data & TBC & TBAN & Taxa adotada \\
\hline $27^{\mathrm{a}}$ & $02 / 09 / 1998$ & $19,00 \%$ & $29,75 \%$ & $24,375 \%$ \\
\hline $28^{\mathrm{a}}$ & $10 / 09 / 1998$ & $19,00 \%$ & $49,75 \%$ & $34,375 \%$ \\
\hline $29^{\mathrm{a}}$ & $07 / 10 / 1998$ & $19,00 \%$ & $49,75 \%$ & $34,375 \%$ \\
\hline $30^{\mathrm{a}}$ & $11 / 11 / 1998$ & $19,00 \%$ & $42,25 \%$ & $30,625 \%$ \\
\hline
\end{tabular}

Portanto, estas quatro observações caracterizaram-se por taxas médias que estiveram acima da TBC e abaixo da TBAN, o que leva a presumir que a simples adoção da TBC (ou da TBAN), nestes casos, não representaria adequadamente a realidade do período. Assim, parece mais verossímil adotar um valor entre estes dois limites - a ideia mais imediata e intuitiva é tomar a média de ambos, como mostrou a Tabela 2 - espelhando assim um pouco mais fidedignamente o ambiente econômico da época. Assim, para os casos listados, tomou-se a média entre TBC e TBAN como representativa da taxa básica de juro.

Por fim, todos os erros-padrão são calculados seguindo o procedimento sugerido por Newey e West (1987), para serem robustos à heterocedasticidade e à correlação serial da amostra, cujas presenças são indicadas pelas estatísticas de Durbin e Watson (DW) e também pelos demais testes expressos nas tabelas do item a seguir.

\section{RESULTADOS}

O primeiro passo é a detecção das observações que possam ser consideradas outliers, ou seja, aquelas cuja influência estatística nos resultados da regressão seja excessivamente maior que as demais; para tanto, retomando-se então (10) e aplicando-se a (8), conforme descrito em 3.1, encontramos cinco observações que se enquadram ao critério, conforme expresso pela distribuição mostrada no Gráfico 1.

Não coincidentemente, tais observações estão ligadas a circunstâncias relevantes da histórica econômica brasileira contemporânea; são elas:

- 30/10/1997: Nesta ocasião eclodiu a crise nos países asiáticos; em decisão extraordinária, o Banco Central retroagiu na decisão regular anterior (de dias antes) e praticamente dobrou a taxa básica de juro, além de outras medidas objetivando a reversão das expectativas então formadas;

- 02 e 10/09/1998: em agosto de 1998 iniciou-se a crise russa (que teve como desfecho sua moratória), disseminando assim uma instabilidade internacio- 
nal que obrigou o Banco Central a implantar um novo pacote de medidas, entre elas a elevação da TBAN. Em particular, a mudança do dia 10 foi de caráter extraordinário, dada a celeridade dos acontecimentos da ocasião;

- 18/01/1999 e 04/03/1999: como já mencionado em 3.2, em 15/01/1999 o Banco Central adotou o regime cambial flutuante. Na reunião seguinte, em 04/03/1999, uma forte subida da taxa básica de juro foi implementada, no intuito de se promover uma reversão das expectativas, dada a deterioração do cenário econômico desde o final de 1998.

Gráfico 1: Distribuição das influências estatísticas obtidas para cada uma das 124 observações, conforme equação (10) aplicada à regressão (8).

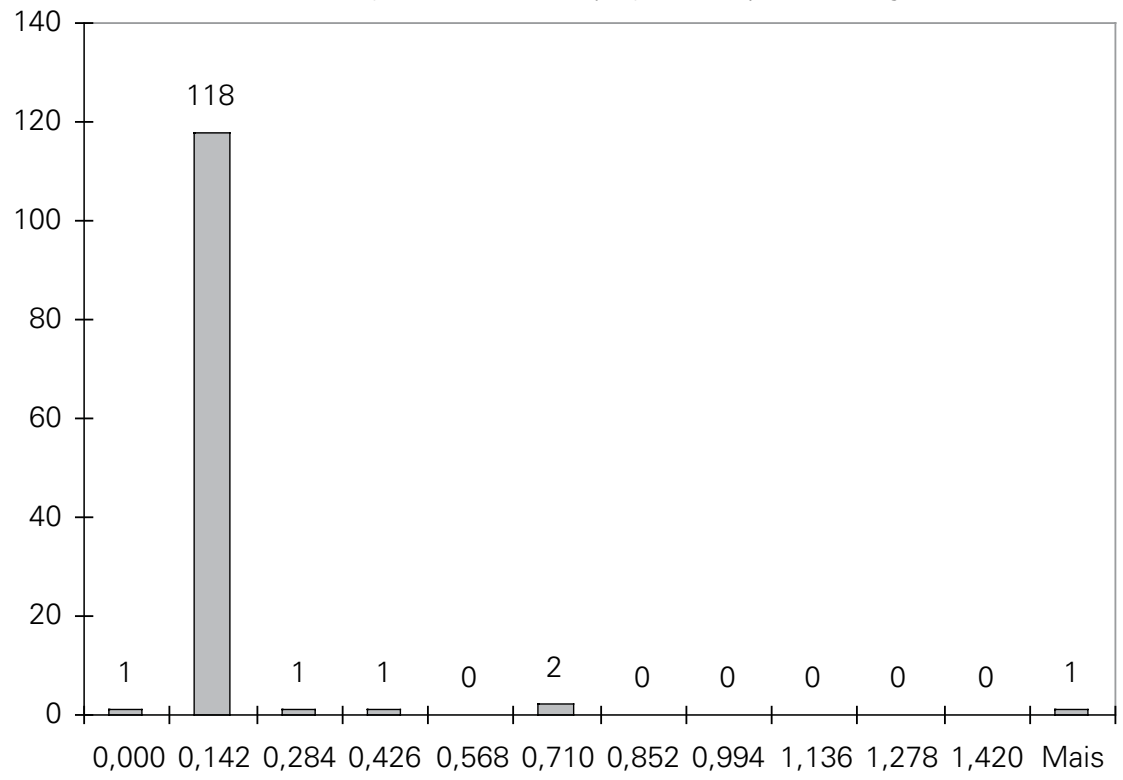

Limite Superior do Intervalo

Conhecidas então as observações de grande influência nos resultados, doravante os testes serão procedidos com dois conjuntos de dados: o primeiro, completo, com todas as observações pertinentes ao estudo em foco; o outro, com as mesmas características, porém tendo estas cinco observações previamente removidas. Isso permitirá (como já aludido) verificar-se a robustez das respostas que obtivermos. Esta sistemática será iniciada então com a apresentação da Tabela 3. 
Tabela 3: Principais estatísticas descritivas das variáveis selecionadas

\begin{tabular}{|l|ccc|ccc|}
\hline \multirow{2}{*}{ Média } & \multicolumn{3}{c|}{ Com Outliers } & \multicolumn{3}{c|}{ Sem Outliers } \\
\cline { 2 - 7 } & $\mathrm{I}^{\mathrm{e}}$ & $\mathrm{I}^{\mathrm{u}}$ & $\Delta \mathrm{P}^{\mathrm{t}}$ & $\mathrm{I}^{\mathrm{e}}$ & $\mathrm{I}^{\mathrm{u}}$ & $\Delta \mathrm{P}_{\mathrm{t}}$ \\
\hline Mediana & $-0,106 \%$ & $-0,026 \%$ & $-0,132 \%$ & $-0,566 \%$ & $0,013 \%$ & $-0,207 \%$ \\
Máximo & $-0,138 \%$ & $0,011 \%$ & $-0,159 \%$ & $-0,166 \%$ & $0,011 \%$ & $-0,211 \%$ \\
Mínimo & $19,813 \%$ & $1,488 \%$ & $12,578 \%$ & $4,708 \%$ & $1,470 \%$ & $4,766 \%$ \\
Desvio Padrão & $-6,578 \%$ & $-4,373 \%$ & $-9,006 \%$ & $-6,578 \%$ & $-1,734 \%$ & $-5,430 \%$ \\
Assimetria & $3,230 \%$ & $0,660 \%$ & $2,382 \%$ & $1,643 \%$ & $0,405 \%$ & $1,930 \%$ \\
Curtose & 3,830879 & $-3,146249$ & 0,655413 & $-1,08452$ & $-0,313406$ & $-0,272712$ \\
№ Observações & 23,77875 & 21,76954 & 9,532345 & 6,665763 & 7,204113 & 2,970585 \\
Jarque-Bera & 124 & 124 & 124 & 119 & 119 & 119 \\
ADF & 2534,038 & 2024,771 & 229,3473 & 89,95685 & 89,58449 & 1,479328 \\
& $-1,2427$ & $-1,1158$ & $-1,3034$ & $-0,5039$ & $-0,9715$ & $-1,0934$ \\
Phillips-Perron & $(-5,57)$ & $(-4,72)$ & $(-5,14)$ & $(-3,89)$ & $(-4,05)$ & $(-4,62)$ \\
& $-0,8942$ & $-1,1772$ & $-1,2572$ & $-0,7385$ & $-1,0474$ & $-1,0780$ \\
& $(-9,81)$ & $(-13,1)$ & $(-14,3)$ & $(-8,16)$ & $(-11,24)$ & $(-11,66)$ \\
\hline
\end{tabular}

Como se vê, nela estão explicitadas as principais estatísticas descritivas das variáveis explicativas de (8), para ambos os conjuntos de dados (com e sem os outliers); conforme esperado, a remoção destes elementos reduz a dispersão dos dados e mitiga valores extremos praticamente nas três variáveis; nota-se ainda uma melhoria nos outros momentos (atenuando a não normalidade), bem como a estacionariedade das três séries geralmente a $1 \%$, indicada pelos testes ADF e Phillips-Perron, a despeito da descontinuidade temporal que elas apresentam (fruto da construção para este estudo de evento).

Passando-se então à análise dos resultados das regressões (8) e (9), apresentamos a Tabela 4, onde se vê primeiramente a baixa significância das variações na meta da taxa básica de juro como variável explicativa para o Índice Bovespa. Nota-se também que o mesmo não se aplica ao teste para as suas componentes: não somente os coeficientes da surpresa são significativos a $1 \%$ e apresentam sinal negativo (conforme era inicialmente esperado e alinhado aos resultados de vários trabalhos para a realidade americana), como são robustos aos outliers; a componente esperada não possui a mesma característica, não sendo significativa na amostra sem as cinco observações. 
Tabela 4: Respostas das variações (a uma taxa de capitalização contínua) do Índice

Bovespa às componentes esperada e não esperada (surpresa) das mudanças na meta da taxa básica de juro ou a resposta do mesmo índice às mudanças na meta sem as componentes. Seguem, respectivamente, as especificações (8) e (9), erros-padrão não robustos à heterocedasticidade ou correlação serial.

\begin{tabular}{|lcc|lcc|}
\hline & $\begin{array}{c}\text { Com } \\
\text { Outliers }\end{array}$ & $\begin{array}{c}\text { Sem } \\
\text { Outliers }\end{array}$ & & $\begin{array}{c}\text { Com } \\
\text { Outliers }\end{array}$ & $\begin{array}{c}\text { Sem } \\
\text { Outliers }\end{array}$ \\
\hline Intercepto & $-0,0017$ & $-0,0017$ & Intercepto & $-0,0012$ & $-0,0020$ \\
& $(-0,94)$ & $(-0,91)$ & & $(-0.57)$ & $(-1.05)$ \\
Mudança não & $-1,8766$ & $-1,3145$ & Resposta à variação & 0,0830 & 0,0183 \\
esperada & $(-6,83)$ & $(-3,06)$ & da taxa básica de juro & $(1.24)$ & $(0.17)$ \\
Mudança esperada & 0,1065 & 0,0438 & & & \\
Breusch-Godfrey & $(1,9)$ & $(0,41)$ & & & \\
Serial Correlation LM & 6,6023 & 3,1897 & Breusch-Godfrey & 10,1002 & 3,2736 \\
White & $(0,04)$ & $(0,2)$ & Serial Correlation LM & $(0.01)$ & $(0.19)$ \\
Heteroskedasticity & 17,4896 & 2,7583 & White & 9,9587 & 1,5305 \\
№ Observações & $(0)$ & $(0,74)$ & Heteroskedasticity & $(0.01)$ & $(0.47)$ \\
DW & 124 & 119 & No Observações & 124 & 119 \\
R $^{2}$ & 2,422 & 2,196 & DW & 2,544 & 2,139 \\
\hline
\end{tabular}

Os testes referem-se à estatística $\boldsymbol{n}^{\boldsymbol{o}}$ obs $\boldsymbol{x} \boldsymbol{R}^{\mathbf{2}}$ e os respectivos valores entre parênteses referem-se às suas probabilidades para não rejeição da hipótese nula (não haver correlação serial e não haver heterocedasticidade, respectivamente). Os valores entre parênteses referentes aos coeficientes da regressão são as estatísticas $\boldsymbol{t}$ obtidas a partir de erros-padrão não robustos à heterocedasticidade e correlação serial.

Os testes corroboram, portanto, a hipótese da eficiência informacional desse mercado, explicando também os resultados não significativos para as variações da meta: se os mercados conseguem antecipar uma parte relevante destas variações, então de fato eles deveriam reagir fracamente a elas.

Constataram-se, portanto, respostas significativas somente às novas informações; não obstante, há que se ter alguma cautela: como se vê na mesma tabela, os testes para correlação serial e heterocedasticidade dos resíduos indicaram a presença de ambos os problemas - especialmente na amostra completa. Deste modo, relembrando a observação feita ao final do item 4, optou-se por empregar o procedimento sugerido por Newey e West (1987), cujos resultados são apresentados na Tabela 5.

A aplicação do procedimento em questão garante erros-padrão robustos à correlação serial e heterocedasticidade dos resíduos, o que permite confirmar as conclusões inferidas a partir da tabela anterior; há que se observar, contudo, que embora a surpresa seja uma variável significativa nas variações do Índice Bovespa, o modelo linear simplificado ora proposto (8) tem baixo poder explanatório, especialmente na amostra que desconsidera os outliers, como demonstrado pelo respectivo coeficiente de determinação $\left(\mathrm{R}^{2}\right)$.

De modo geral, as causas para tal são, possivelmente, uma especificação inadequada (variáveis relevantes ausentes, por exemplo), ou uma não linearidade das 
variáveis empregadas, ou até mesmo uma não linearidade da própria regressão a ser usada. Não obstante, o interesse central do trabalho é estudar a significância das variáveis independentes para explicar a dependente, e não propriamente promover-se uma otimização ou excelência no ajustamento da equação regredida.

Tabela 5: Respostas das variações (a uma taxa de capitalização contínua) do Índice Bovespa às componentes esperada e não esperada (surpresa) das mudanças na meta da taxa básica de juro, de acordo com a especificação (8).

\begin{tabular}{|l|c|c|}
\hline & Amostra Completa & Excluindo outliers \\
\hline Intercepto & $-0,0017$ & $-0,0017$ \\
\hline & $(-1,14)$ & $(-1,07)$ \\
\hline Mudança não esperada & $-1,8766$ & $-1,3145$ \\
\hline & $(-4,19)$ & $(-4,32)$ \\
\hline Mudança esperada & 0,1065 & 0,0438 \\
\hline & $(1,54)$ & $(0,45)$ \\
\hline No. Observações & 124 & 119 \\
\hline DW & 2,42 & 2,20 \\
\hline$R^{2}$ & 0,313 & 0,081 \\
\hline
\end{tabular}

Os valores entre parênteses referentes aos coeficientes da regressão são as estatísticas $\boldsymbol{t}$ obtidas a partir dos erros-padrão de Newey-West (1987) robustos à heteroce-dasticidade e correlação serial.

\section{QUEBRAS ESTRUTURAIS E ASSIMETRIAS NAS RESPOSTAS}

Conforme observado no item 3.2, a adoção da taxa básica de juro expressa ao ano-over (252 dias úteis) em 1998, a flexibilização cambial de 1999 e o conjunto de mudanças em caráter extraordinário (em contraposição às oriundas de reuniões regulares) introduziram modificações na dinâmica dos mercados; dessa maneira, o passo seguinte será verificar como tais "perturbações" afetam o objeto deste estudo - é o que mostra a tabela 6 , onde as possíveis fontes de quebra estrutural supracitadas e as influências devidas ao contexto das decisões tomadas são testadas individualmente, segundo a especificação (11).

Assim, os resultados mostram que as fontes de quebra ou as influências de contexto são significativas desde que consideradas as amostras completas; para estas últimas em particular, vê-se que as reuniões extraordinárias apresentam respostas mais intensas, enquanto que as respostas das ordinárias são mais brandas, o que é um resultado esperado, dado que mudanças decorrentes de reuniões não previstas deveriam gerar, tal como observado, reações mais pronunciadas.

No entanto, removidos os outliers, vê-se que não há robustez nas conclusões supra, exceção feita somente à adoção de taxas em expressão anual — esta dummy pareceu absorver a significância da surpresa propriamente dita. No mais, são re- 
sultados concordantes com o anteriormente visto: componente esperada com baixa significância e a surpresa, frequentemente significativa a níveis de $1 \%$ a $5 \%$.

Quanto aos resultados obtidos para a adoção de taxas em expressão anual, tendo em vista que se trata de uma variável dummy interativa que, aparentemente, deslocou a significância da surpresa propriamente dita para si e que são as 105 observações mais recentes e consecutivas da amostra que respondem por esta característica (das 124 da amostra completa), entendemos que o subconjunto por elas formado pode ser excessivamente representativo do todo - desse modo, a interação entre esta dummy e a surpresa poderia, na regressão, representar uma proxy da própria surpresa, o que explicaria tais resultados. Logo, no que se refere a este evento e dada esta ambiguidade, é necessário observar-se que, à luz do exposto, não se pode ainda ser conclusivo sobre este tema.

Tabela 6: Respostas das variações do Índice Bovespa (a uma taxa de capitalização contínua) às componentes esperada e não esperada (surpresa) das

mudanças na meta da taxa básica de juro. Inclui a introdução de variáveis dummy interativas com a surpresa para captar a importância de: mudança cambial de 1999 (após 15/01/1999, dummy = 1 ou, caso contrário, zero); adoção de taxas

anuais (após 02/01/1998, dummy = 1 ou, caso contrário, zero) e mudanças regulares (se não são as relacionadas na tabela 1, dummy = 1; caso contrário, zero), todas de acordo com o exposto em 3.2 e a especificação (11).

\begin{tabular}{|c|c|c|c|c|c|c|}
\hline & $\begin{array}{l}\text { Amostra } \\
\text { Completa }\end{array}$ & $\begin{array}{c}\text { Excluindo } \\
\text { outliers }\end{array}$ & $\begin{array}{l}\text { Amostra } \\
\text { Completa }\end{array}$ & $\begin{array}{c}\text { Excluindo } \\
\text { outliers }\end{array}$ & $\begin{array}{l}\text { Amostra } \\
\text { Completa }\end{array}$ & $\begin{array}{l}\text { Excluindo } \\
\text { outliers }\end{array}$ \\
\hline \multirow{2}{*}{ Intercepto } & $-0,0015$ & $-0,0017$ & $-0,0024$ & $-0,0019$ & $-0,0022$ & $-0,0018$ \\
\hline & $(-1,01)$ & $(-1,12)$ & $(-1,62)$ & $(-1,27)$ & $(-1,46)$ & $(-1,12)$ \\
\hline \multirow{2}{*}{ Mudança não esperada } & $-2,2822$ & $-0,8412$ & $-2,5123$ & $-1,8112$ & 0,3862 & 0,0252 \\
\hline & $(-4,09)$ & $(-1,7)$ & $(-6,59)$ & $(-2,29)$ & $(0,82)$ & $(0,05)$ \\
\hline \multirow{2}{*}{ Mudança esperada } & 0,0979 & 0,0462 & 0,0708 & 0,0571 & 0,0410 & 0,0338 \\
\hline & $(1,22)$ & $(0,48)$ & $(1,02)$ & $(0,62)$ & $(0,77)$ & $(0,34)$ \\
\hline $\begin{array}{l}\text { Mudança não esperada } \\
\text { x Dummy Cambial }\end{array}$ & $\begin{array}{l}0,9096 \\
(1,54)\end{array}$ & $\begin{array}{r}-0,7168 \\
(-0,96)\end{array}$ & & & & \\
\hline $\begin{array}{l}\text { Mudança não } \\
\text { esperada x Dummy } \\
\text { Reuniões Regul. }\end{array}$ & & & $\begin{array}{l}1,1301 \\
(2,08)\end{array}$ & $\begin{array}{l}0,7241 \\
(0,72)\end{array}$ & & \\
\hline $\begin{array}{l}\text { Mudança não esperada } \\
\text { x Dummy taxa anual }\end{array}$ & & & & & $\begin{array}{r}-2,4772 \\
(-3,16)\end{array}$ & $\begin{array}{r}-1,4466 \\
(-2,47)\end{array}$ \\
\hline № Observações & 124 & 119 & 124 & 119 & 124 & 119 \\
\hline DW & 2,36 & 2,20 & 2,35 & 2,20 & 2,40 & 2,18 \\
\hline$R^{2}$ & 0,329 & 0,086 & 0,335 & 0,086 & 0,335 & 0,087 \\
\hline
\end{tabular}

Os valores entre parênteses referentes aos coeficientes da regressão são as estatísticas $\mathbf{t}$ obtidas a partir dos erros-padrão de Newey-West (1987) robustos à heterocedasticidade e correlação serial. 
Tabela 7: Respostas das variações do Índice Bovespa (a uma taxa de capitalização contínua) às componentes esperada e não esperada (surpresa) das mudanças na meta da taxa básica de juro. Inclui a introdução de variáveis dummy interativas com a surpresa para verificação de possíveis assimetrias dummy $=1$ se o movimento ocorre na direção selecionada ou, caso contrário, zero), de acordo com a especificação (11).

\begin{tabular}{|c|c|c|c|c|c|c|c|c|}
\hline & $\begin{array}{l}\text { Amostra } \\
\text { Completa }\end{array}$ & $\begin{array}{c}\text { Excluindo } \\
\text { outliers }\end{array}$ & $\begin{array}{l}\text { Amostra } \\
\text { Completa }\end{array}$ & $\begin{array}{c}\text { Excluindo } \\
\text { outliers }\end{array}$ & $\begin{array}{l}\text { Amostra } \\
\text { Completa }\end{array}$ & $\begin{array}{l}\text { Excluindo } \\
\text { outliers }\end{array}$ & $\begin{array}{l}\text { Amostra } \\
\text { Completa }\end{array}$ & $\begin{array}{c}\text { Excluindo } \\
\text { outliers }\end{array}$ \\
\hline \multirow[t]{2}{*}{ Intercepto } & $-0,0015$ & $-0,0017$ & $-0,0013$ & $-0,0016$ & $-0,0016$ & $-0,0014$ & $-0,0015$ & $-0,0018$ \\
\hline & $(-1,02)$ & $(-1,11)$ & $(-0,85)$ & $(-1,07)$ & $(-1,02)$ & $(-0,9)$ & $(-1,1)$ & $(-1,16)$ \\
\hline \multirow{2}{*}{$\begin{array}{l}\text { Mudança não } \\
\text { esperada }\end{array}$} & $-1,2674$ & $-1,3531$ & $-2,5301$ & $-1,5731$ & $-1,8612$ & $-1,2145$ & $-1,6156$ & $-1,1045$ \\
\hline & $(-6,16)$ & $(-4,84)$ & $(-5,53)$ & $(-1,79)$ & $(-3,95)$ & $(-3,73)$ & $(-4,66)$ & $(-3,18)$ \\
\hline \multirow[t]{2}{*}{ Mudança esperada } & 0,0954 & 0,0444 & 0,0947 & 0,0441 & 0,1065 & 0,0476 & 0,1333 & 0,0643 \\
\hline & $(1,13)$ & $(0,45)$ & $(1,13)$ & $(0,46)$ & $(1,53)$ & $(0,49)$ & $(1,98)$ & $(0,68)$ \\
\hline $\begin{array}{l}\text { Mudança não } \\
\text { esperada x Dummy } \\
\text { aumento da taxa }\end{array}$ & $\begin{array}{r}-1,2668 \\
(-2,64)\end{array}$ & $\begin{array}{c}0,2796 \\
(0,21)\end{array}$ & & & & & & \\
\hline $\begin{array}{l}\text { Mudança não } \\
\text { esperada x Dummy } \\
\text { redução da taxa }\end{array}$ & & & $\begin{array}{l}1,3354 \\
(3,08)\end{array}$ & $\begin{array}{c}0,3304 \\
(0,36)\end{array}$ & & & & \\
\hline $\begin{array}{l}\text { Mudança não } \\
\text { esperada x Dummy } \\
\text { inatividade }\end{array}$ & & & & & $\begin{array}{r}-0,5207 \\
(-0,49)\end{array}$ & $\begin{array}{r}-1,2589 \\
(-1,46)\end{array}$ & & \\
\hline $\begin{array}{l}\text { Mudança não } \\
\text { esperada x Dummy } \\
\text { reversão }\end{array}$ & & & & & & & $\begin{array}{r}-3,2509 \\
(-3,71)\end{array}$ & $\begin{array}{r}-1,9443 \\
(-1,42)\end{array}$ \\
\hline № Observações & 124 & 119 & 124 & 119 & 124 & 119 & 124 & 119 \\
\hline DW & 2,35 & 2,19 & 2,36 & 2,20 & 2,43 & 2,20 & 2,35 & 2,25 \\
\hline $\mathrm{R}^{2}$ & 0,344 & 0,082 & 0,347 & 0,082 & 0,314 & 0,086 & 0,368 & 0,096 \\
\hline
\end{tabular}

Respostas das variações do Índice Bovespa (a uma taxa de capitalização contínua) às componentes esperada e não esperada (surpresa) das mudanças na meta da taxa básica de juro. Inclui a introdução de variáveis dummy interativas com a surpresa para verificação de possíveis assimetrias $($ dummy $=1$ se o movimento ocorre na direção selecionada ou, caso contrário, zero), de acordo com a especificação (11).

No que se refere à hipótese de que os mercados possam reagir diferentemente conforme a direção das mudanças na taxa básica de juro, como se vê na Tabela 7 , novamente se confirmam resultados previamente conhecidos a respeito da componente esperada e também da surpresa (estes últimos robustos); verificou-se também significância para aumentos, reduções e reversões na taxa desde que considerados na amostra completa - removidos os outliers, a significância sucumbe; portanto, dada a falta de robustez dos resultados (indicações contraditórias pelas duas amostras), não se pode afirmar que os mercados respondam assimetricamente às ações de política monetária, ou seja, que as intensidades das respostas sejam significati- 
vamente ligadas às direções das decisões tomadas pelo COPOM para mudanças na taxa básica de juro.

\section{CONCLUSÕES}

Tendo como base conceitual a hipótese dos mercados eficientes, foi definida uma medida que captasse a surpresa gerada nos mercados a cada decisão do COPOM. Estas medidas são eminentemente baseadas em dados do mercado de futuros DI 1 dia, cuja frequência, liquidez e similaridade à taxa SELIC fazem desses instrumentos não somente ferramentas úteis, mas também versáteis para explorações sob diferentes horizontes e necessidades.

De posse desse conjunto de informações assim construído - medidas calculadas, mais as variações do Índice Bovespa, formou-se o banco de dados que respaldou o estudo de evento aqui realizado. Assim, como importantes resultados, constatou-se que o mercado acionário reage fracamente às variações diretas na meta da taxa básica de juro - uma consequência da capacidade do mesmo em se antecipar às decisões do COPOM; entretanto, verificou-se uma resposta significativa e robusta às variações inesperadas (tal como previsto pela hipótese da eficiência informacional dos mercados), estimando-se que a cada ponto percentual de incremento não esperado na meta da taxa básica esteja associada uma queda média de $1,3 \%$ do Índice Bovespa.

Uma descoberta adicional foi a de que relevantes eventos contemporâneos de ruptura econômica pareceram não interferir de modo robusto neste padrão de respostas às surpresas, o mesmo se observando com relação à direção tomada pelas mudanças; a exceção a ser citada refere-se à adoção de taxas de juros em expressão anual - como mencionado no item 5 , os dados e métodos aqui empregados não permitiram uma posição conclusiva sobre o tema, sendo necessários estudos complementares que ajudem a elucidar a questão.

É importante ressalvar, contudo, que os resultados obtidos originaram-se de um estudo de evento cujo caráter é essencialmente empírico. Assim, é necessário desenvolverem-se modelos nos quais se possa não somente analisar o papel destas variáveis na formação dos preços, como também investigar outras implicações e desdobramentos teóricos; outro fato a se destacar é que estas conclusões repousam em uma diretriz inicialmente assumida: a janela de evento selecionada é de um dia útil - conforme sugerido por Campbell et al. (1996), o estreitamento desta janela, desde que conceitualmente factível, é recomendável sempre e pode ajudar a preservar a necessária ortogonalidade entre os resíduos das regressões e outras variáveis, envolvidas ou não no estudo.

Deve, também, ser observado que este estudo tem como premissa implícita que os mercados reagem às decisões do COPOM, e não o oposto (assume-se que política monetária é exógena em relação aos mercados); não obstante tal assunção, ao tratarmos da questão da ortogonalidade supra referida (bem como em 3.1), não se pode descartar a possibilidade de haver eventuais ocorrências de simultaneidade 
nas respostas de ambos os agentes (governo e mercados) a certos eventos ou tendências verificadas na economia.

Embora o critério adotado quanto à janela do estudo de evento ajude a minimizar sobremaneira tal problema, evidentemente houve situações em que as reações foram conjuntas - não coincidentemente, esta parece ser a característica dos cenários de stress anteriormente descritos que deram origem às cinco observações classificadas como outliers - note-se como esta constatação ratifica a importância da duplicidade nos testes conduzidos, com e sem tais observações, originalmente visando-se tão somente robustez nos resultados.

De qualquer modo, Bernanke e Kuttner (2005) observaram que esse problema, assim como outras características do estudo de evento, levam a inferências qualitativamente corretas quanto às respostas, a despeito de se ter detectado um viés que tende a subestimá-las em suas intensidades. Assim, estes problemas não invalidam as conclusões obtidas ao longo deste trabalho - embora se deva alertar que os efeitos então estudados podem ser ainda mais pronunciados que os ora estimados.

Por conta disto, recomendam-se algumas sugestões para se lidar com a questão: Gürkaynak, Sack e Swanson (2004), por exemplo, empregaram dados intraday como forma de mitigar ainda mais o problema (embora isto constitua um obstáculo de difícil transposição na realidade brasileira, como já mencionado anteriormente); é possível tentar a abordagem de Rigobon e Sack (2004), a qual se vale de estimadores que exploram a heterocedasticidade introduzida por ações exógenas de política monetária; há ainda a possibilidade explorada por Poole, Rashe e Thornton (2002) dos erros de medidas nos regressores e a proposição de uma especificação modificada para corrigir o problema.

Por fim, entendemos que os próximos passos a serem seguidos consistirão na proposição de modelos que ajudem a expandir os horizontes deste assunto, bem como novos testes econométricos segundo as diferentes abordagens sugeridas (ou outras não tratadas aqui) que podem se constituir em campo fértil para novas contribuições a se somarem ao mainstream já estabelecido.

\section{REFERÊNCIAS BIBLIOGRÁFICAS}

BANCO CENTRAL DO BRASIL. Atas das Reuniões do COPOM; disponível em www.bcb.gov.br. BERNANKE, Ben; KUTTNER, Kenneth. (2005) "What explains the stock market's reaction to Federal Reserve Policy?”, Journal of Finance, 60 (3): 1221-1257.

BOMFIM, Antulio N.; REINHART, Vincent R. (2000) "Making news: Financial market effects of federal reserve disclosure practices", Finance and Economics Discussion, Series Working Paper \#2000-14, Federal Reserve Board of Governors.

BOMFIM, Antulio N. (2003) "Pre-announcement effects, news effects, and volatility: monetary policy and the stock market", Journal of Banking and Finance, 27: 133-151.

CAMPBELL, John Y.; LO, Andrew W.; MACKINLAY, A.C. (1996) The Econometrics of Financial Markets, Princeton University.

COOK, T.; HAHN, T. (1989) "The effect of changes in the federal funds rate target on market interest rates in the 1970s", Journal of Monetary Economics, 24: 331-352. 
CRAINE, Roger; MARTIN, Vance (2003), “Monetary policy shocks and security market responses”, Working Paper, University of California at Berkeley.

DEWBRE, J. H. (1981) “Interrelationships between spot and futures markets: some implications of rational expectations, American Journal of Agricultural Economics, v. 63: 925-933.

GARCIA, Márcio G. P.; LA ROCQUE, Eduarda C. O. (1994) "Mercado futuro de taxas de juros no Brasil: Especificidades teóricas e empíricas do mercado de DI futuro", Texto para Discussão No 318, Pontifícia Universidade Católica do Rio de Janeiro, Departamento de Economia.

GONÇALVES, Carlos E.S.; GUIMARÃES, Bernardo (2005) "Monetary policy and the exchange rate in Brazil”, Working Paper, disponível em http://personal.lse.ac.uk/guimarae.

GÜRKAYNAK, Refet S.; SACK, Brian P.; SWANSON, Eric T. (2004) "The effects of monetary policy on asset prices: an intraday event-study analysis", Working Paper, Board of Governors of the Federal Reserve System.

GÜRKAYNAK, Refet S. (2005) "Using federal funds futures contracts for monetary policy Analysis." Working Paper, Board of Governors of the Federal Reserve System (U.S.) Washington, D.C.: Divisions of Research \& Statistics and Monetary Affairs.

HONDA, Yuzo; KUROKI, Yoshihiro (2006) "Financial and capital markets' responses to changes in the central bank's target interest rate: the case of Japan", The Economic Journal, 116: 812-842.

KUTTNER, Kenneth N. (2001), "Monetary policy surprises and interest rates: evidence from the Fed funds futures market", Journal of Monetary Economics, 47: 523-544.

LUCAS Jr. R. E.; SARGENT, T. (1996) “After Keynesian macroeconomics, in Miller, P. J. (org.) The Rational Expectations Revolutions: Readings from the Front Line. [S.1]: Massachussetts Institute of Technology, pp. 5-30.

NEWEY, Whitney; WEST, Kenneth (1987) “A simple, positive semi-definite, heteroskedasticity and autocorrelation consistent covariance matrix, Econometrica, 55: 703-708.

PEROBELLI, Fernanda F.C.; PEROBELLI, Fernando S.; ARBEX, Marcelo A. (2000) "Expectativas racionais e eficiência informacional: análise do mercado acionário brasileiro no período 1997. 1999", Revista de Administração Contemporânea. 4 (2), maio/ago.

POOLE, William; RASCHE, Robert; THORNTON, Daniel (2002) "Market anticipations of monetary policy actions", Federal Reserve Bank of St. Louis Review, 84: 65-93.

REINHART, Vincent; SIMIN, Timothy (1997) "The market reaction to federal reserve policy action from 1989 to 1992", Journal of Economics and Business. 49 (2): 149-168.

RIGOBON, Roberto; SACK, Brian (2004) “The impact of monetary policy on asset prices”, Finance and Economics Discussion Series 2002-4, Board of Governors of the Federal Reserve System.

ROBITAILLE, Patrice; ROUSH, Jennifer. (2005) "How do Brazilian yield spreads and stock prices respond to FOMC actions and U.S. macroeconomic data announcements?", Working Paper, Federal Reserve Bank.

ROLEY, V. Vance; SELLON Jr., Gordon H. (1995) “Monetary policy actions and long-term interest rates. Federal Reserve Bank of Kansas City, Economic Review, 80: 73-89.

(1998a) "The response of interest rates to anticipated and unanticipated policy actions", Working Paper, University of Washington.

. (1998b) "Market Reaction to monetary policy nonannouncements", Working Paper, Federal Reserve Bank of Kansas City.

SMIRLOCK, M., and YAWITZ, J. (1985) "Asset returns, discount rate changes, and market efficiency", Journal of Finance, 40: 1141-1158.

TABAK, Benjamim M.; TABATA, Alicia (2004a) "Surpresas na política monetária e a estrutura a termo da taxa de juro brasileira”, Economia Aplicada, 8 (3): 383-399.

TABAK, Benjamim M.; TABATA, Alicia. (2004b) “Testando o conteúdo informacional das decisões de política monetária”. Quarto Encontro Brasileiro de Finanças.

THORBECKE, W.; ALAMI, T. (1994) "The effect of changes in the federal funds rate target on stock prices in the 1970s", Journal of Economics and Business, 46: 13-19.

THORBECKE, W. (1997) “On stock market returns and monetary policy”, Journal of Finance, 52 (2): 635-654. 